\title{
Diez mitos en torno al consentimiento informado
}

\section{Ten myths about informed consent}

\author{
P. Simón
}

\section{RESUMEN}

El autor realiza una revisión de los diez mitos que, a su juicio, configuran la visión de muchos profesionales sobre el consentimiento informado (CI). Visión, por otra parte, poco precisa y de baja calidad ética y jurídica. Los mitos son: 1. El CI es algo extraño a los deberes morales de los médicos. 2. El CI consiste en conseguir que los pacientes firmen el formulario en el que autorizan la realización de una intervención. 3. La buena práctica clínica consiste en realizar bien los actos clínicos desde el punto de vista científico-técnico; el $\mathrm{CI}$ no tiene que ver con esto. 4 . El fundamento ético del CI es el principio de autonomía. 5. La obtención del CI es una obligación de los profesionales que realizan las intervenciones, pero no de los que las indican. 6. El CI no tiene ningún sentido porque la mayoría de los pacientes no desean ser informados. 7. Los pacientes tienen derecho a rechazar un tratamiento, pero sólo antes de que se les aplique. Una vez iniciado éste, los profesionales no pueden retirarlo. 8. La evaluación de la capacidad de los pacientes para decidir es responsabilidad de los psiquiatras. 9. El respeto a las exigencias del CI puede satisfacerse sin que las organizaciones sanitarias inviertan recursos adicionales en ello. 10. La obtención del CI es una responsabilidad de los médicos, pero no de la enfermería. En la conclusión se insiste en la importancia de desvelar el discurso oculto en estos mitos para poder cambiar las prácticas sobre el CI en nuestras organizaciones sanitarias.

Palabras clave. Consentimiento informado, bioética, derecho, capacidad.

\begin{abstract}
The author reviews ten myths that, in his opinion, make up the perspective of many professionals on informed consent (IC). A viewpoint, on the other hand, that is imprecise and of low ethical and juridical quality. The myths are: 1 . IC is something foreign to the moral duties of doctors. 2. IC consists in getting the patients to sign the form authorising an intervention. 3 . Good clinical practice consists in carrying out clinical actions well, from a scientific-technical point of view; IC has nothing to do with this. 4 . The ethical foundation of IC is the principle of autonomy. 5. Obtaining IC is an obligation of the professionals who carry out interventions, but not of those who prescribe them. 6 . IC is meaningless because the majority of patients do not want to be informed. 7 . Patients have the right to refuse a course of treatment, but only before it is applied to them. Once it is started, the professionals cannot withdraw it. 8. The evaluation of the capacity of the patients to make decisions is the responsibility of the psychiatrists. 9. Respect for the requirements of IC can be satisfied without the health organisations investing additional resources in this. 10. Obtaining IC is a responsibility of the doctors but not of the nursing staff. The conclusion insists on the importance of exposing the hidden discourse of these myths in order to be able to change the practices concerning IC in our health organisations.
\end{abstract}

Key words. Informed consent. Bioethics. Law. Capacity.

An. Sist. Sanit. Navar. 2006; 29 (Supl. 3): 29-40.

Profesor de la Escuela Andaluza de Salud Pública. Granada

\section{Correspondencia:}

E-mail: pablo.simon.easp@juntadeandalucia.es 
"Todo ha cambiado y nada ha cambiado" ("Everything's changed, and nothing's changed"). Esta era la síntesis diagnóstica que realizaban Faden y Beauchamp, en su crucial y célebre libro sobre consentimiento informado, acerca del estado de desarrollo de dicha teoría en EE.UU. en 1986 -hace por tanto 20 años-1. "Todo ha cambiado" porque las estructuras sociales, culturales, políticas, legales y profesionales que amparaban el paternalismo tradicional habían prácticamente desaparecido, y habían sido sustituidas por otras que propiciaban la participación de los pacientes en la toma de decisiones sanitarias. Pero "nada ha cambiado" porque en el fondo, muchos de los sanitarios norteamericanos habían claudicado en la forma por motivos estrictamente legales, pero en el fondo no habían asumido el cambio de mentalidad ética y profesional que llevaba pareja la idea del consentimiento informado, y por tanto sus verdaderas actitudes y querencias seguían siendo en realidad las del paternalismo.

La hipótesis diagnóstica que barajaré a lo largo de este trabajo con respecto a la situación de la teoría del consentimiento informado en nuestro país en el día de hoy es muy similar a la que describían Faden y Beauchamp en 1986. Quizás no sea justo que el diagnóstico sea tan pesimista como el suyo; indudablemente muchas cosas han cambiado en nuestro país en estos años, y lo han hecho para bien, pero quizás no lo han hecho todo lo que podían y debían. "Todo ha cambiado respecto al consentimiento informado en España, pero, en el fondo, poco ha cambiado".

Este "cambio con poco cambio" puede desvelarse a través de los mitos que configuran el "saber" y, consiguientemente, el "no-saber" de los profesionales españoles en torno al concepto de "consentimiento informado". Este trabajo es un ejercicio de indagación en torno a algunos de estos mitos, en concreto de diez de ellos..., y contra ellos, claro.

MITO 1. El consentimiento informado es un invento caprichoso y arbitrario de los abogados, jueces y legisladores, totalmente extraño a los deberes morales de los médicos y que no hace sino entorpecer el normal ejercicio de la medicina.

Respuesta. Este mito encierra una paradoja: que es al mismo tiempo verdadero y falso. Todo depende de lo que se entienda por "deberes morales de los médicos" y por "normal ejercicio de la medicina". Si la perspectiva adoptada es la de la tradición médica, la que se despliega entre el siglo III a. d. C. y la primera mitad del siglo XX, entonces la afirmación es verdadera. Si la perspectiva que se adopta es la que a partir de mediados del siglo XX ha ido configurando, poco a poco, una nueva manera de entender lo que es hacer "buena medicina", entonces la afirmación es falsa.

Explicación. Obviamente no se puede repasar aquí todo el desarrollo histórico de este proceso de cambio tal y como se ha hecho en otro lugar². Sólo resaltaré lo que resulta crucial; a saber, que la "idea" de consentimiento informado no surge en el campo de la medicina sino en el de la filosofía política. Aparece en escena en los albores de la Modernidad -en medio de las luchas entre protestantes y católicos, en pleno siglo XVI- y desde ahí, y sólo desde ahí, llegará al campo de la medicina casi cuatro siglos más tarde. La idea del "consentimiento informado" no es por tanto un "invento" de jueces y legisladores, sino la piedra clave de la organización de las sociedades modernas.

La idea que late en el "consentimiento informado" es que las relaciones humanas, sean del tipo que sean, públicas o privadas, no pueden basarse en una concepción vertical donde una parte detenta todo el poder y la otra sólo puede obedecer pasivamente. Este ejercicio monopolístico del poder se basa en la pretendida superioridad del conocimiento por parte de aquellos que lo detentan. El médico, el gobernante y el sacerdote están por encima de los demás porque saben qué hay que hacer para mantener el orden en los diferentes ámbitos de la realidad. Ellos son los garantes de la salud física, social y espiritual de la comunidad. Ellos cuidan de los demás como el "buen padre" cuida de sus hijos pequeños, ignorantes, indefensos, incapaces. El contenido de lo que sea 
"hacer el bien", tanto en el sentido técnico como en el moral, es definido unilateralmente por este grupo de personas dotadas de un conocimiento superior.

La Modernidad, ese largo proceso histórico que ha durado cinco siglos de nuestra historia, no es, en el fondo, más que el costoso proceso de deslegitimación y destrucción de todo este modelo de pensamiento. Los súbditos se convertirán ahora en ciudadanos con derechos, sujetos con libertad de conciencia para decidir qué religión profesar y qué estructura política construir. La autodeterminación de las personas, el ejercicio de la propia soberanía sobre el cuerpo y la mente, y la imposibilidad de recortarla salvo cuando perjudique a otros -como dirá Stuart Mill dos siglos después- se convierte en la clave moral del proyecto sociopolítico de la Modernidad.

El aterrizaje de estas ideas en el ámbito de la relación médico-paciente fue tardío. La vulnerabilidad del enfermo y su dependencia respecto al saber médico en algo tan sensible como la vida y la salud, hicieron este proceso especialmente largo. El fenómeno comenzó en los albores del siglo $\mathrm{XX}$ en los EE.UU. y poco a poco se ha ido extendiendo por todas las democracias de corte occidental. Los pacientes comenzaron a "decir": "somos ciudadanos con derechos y las relaciones sociales que establecemos se basan en el libre consentimiento mutuo; por tanto, no vemos la razón por la que al entrar en un hospital dejamos de tener la consideración de ciudadanos y se nos obliga a establecer unas relaciones basadas en la desinformación y el sometimiento". Y también "decían": "el concepto de salud y enfermedad no es sólo algo objetivo, determinado unilateralmente por los médicos, sino también una experiencia subjetiva donde los valores del afectado son claves para determinar lo que le conviene". El médico ya no puede saber por sí solo en qué consiste "hacer el bien" al paciente. La voz de éste es imprescindible; sin ella no hay actos clínicos correctos. Los médicos ya no son los señores de la vida y la muerte, la salud y la enfermedad
Así, durante el siglo XX, los pacientes reclamaron que los médicos reconocieran la necesidad de replantear totalmente el paternalismo de las relaciones clínicas porque no respondía al desarrollo moral de la sociedad, sino a esquemas totalmente superados en la vida sociopolítica. La tristeza es que este proceso de cambio haya tenido que ser, en buena medida, impuesto al colectivo médico mediante sentencias judiciales y cambios legislativos. Esto ha sido muy traumático para los profesionales y generado conductas aberrantes como la medicina defensiva -práctica de bajísima calidad científica y éticao percepciones "victimistas" en las que todos los demás son agresores potenciales: pacientes, familias, jueces, equipos directivos, políticos, etc.

Lo ideal hubiera sido, lógicamente, que la profesión médica hubiera detectado el cambio histórico, sociológico, político y moral que se estaba gestando a su alrededor y hubiera impulsado desde dentro ese imparable cambio de paradigma de las relaciones sanitarias. Es cierto que las sociedades científicas y los colegios de médicos lo han ido haciendo poco a poco, pero de manera muy lenta. De hecho, el cambio no se ha producido todavía en unas instituciones clave: las facultades de medicina. El modelo de conducta que, explícita o implícitamente, se enseña en ellas es, con demasiada frecuencia, o bien el del paternalismo clásico benefactor, o bien el de una descarnada mentalidad científico-tecnológica para la que el paciente es un mero objeto de estudio y práctica.

MITO 2. El consentimiento informado consiste en conseguir que los pacientes firmen el formulario escrito en el que autorizan la realización de una intervención.

Respuesta. Falso. El consentimiento informado es, básicamente, un proceso verbal que se despliega en el interior del acto clínico formando parte de él. Así lo dice de forma reiterada la Ley 41/2002 básica de autonomía de los pacientes ${ }^{3}$. El formulario de consentimiento informado es una de las herramientas que el profesional puede utilizar para proporcionar información a los pacientes y registrar su con- 
sentimiento, pero ni siquiera es de las más importante porque su uso está limitado a determinadas intervenciones. Más importante es, sin duda, como práctica general, el registro en la historia clínica.

Explicación. La reducción del consentimiento informado al ritual burocrático de la firma de los formularios es quizás una de sus más graves lacras. Ya en 1978, James M. Vaccarino -uno de los más prestigiosos editorialistas del New England Journal of Medicine- lo denunciaba lúcidamente desde las páginas de esta revista (Tabla 1). Parece mentira que, casi 30 años después, sus palabras mantengan toda su actualidad, al menos en nuestro país.

Exactamente lo mismo puede decirse del análisis de los dos modelos básicos de obtención del consentimiento informado que publicaron hace casi 20 años, en 1988, otros tres autores prestigiosos: Paul S. Appelbaum, Charles Lidz y Alan Meisel ${ }^{4}$. En lo que ellos llamaron el modelo puntual (event model) de consentimiento informado, el formulario es el verdadero protagonista y la óptica es básicamente legal. Bajo este prisma lo importante es diseñar formularios que cumplan los requisitos lega- les en cuanto a cantidad y calidad de información y dárselos a leer al paciente para que los firme. Una vez se ha conseguido esto (la firma), se ha cumplido el objetivo prioritario: asegurar, en la medida de lo posible, la protección legal del médico.

Por el contrario, en el modelo progresivo (process model) la principal función del formulario es servir de guía en el proceso de información y de diálogo con el médico. Lo sustantivo es el proceso oral de comunicación y no el formulario, que es sólo un medio de apoyo. Sin duda el formulario debe de estar adecuadamente construido desde el punto de vista legal para favorecer la protección del médico, pero esta perspectiva es secundaria en esta forma de entender el consentimiento.

Lo que late en el fondo del modelo puntual es, a mi modo de ver, simple y llanamente el paternalismo de antaño. Lo único que sucede es que éste se ha visto obligado a incorporar a sus formas de trabajo -por ser un requisito legal- una de las herramientas desarrolladas por la nueva filosofía del consentimiento informado, pero sin cambiar en el fondo ninguna de sus convicciones éticas. Por eso el modelo

Tabla 1. Los problemas de los formularios escritos de consentimiento informado según Vaccarino ${ }^{1}$.

\begin{abstract}
"Al ser incapaces de distinguir entre el consentimiento y su documentación, los profesionales del Derecho han precipitado una enorme confusión entre los médicos en lo relativo al consentimiento informado, esto es, que si un formulario está firmado, el consentimiento informado ha sido obtenido.

En última instancia, el formulario de consentimiento debe de ser tenido por lo que es: nada más que un indicio de que el consentimiento informado fue obtenido.

En realidad la única manera de obtener el consentimiento informado es mediante una conversación. A medida que los tribunales han dictaminado cuestiones tales como lo que debe de decírsele al paciente y la extensión, gravedad y probabilidad del riesgo, en un intento de desarrollar un formulario de consentimiento ideal, los médicos han hecho evolucionar los formatos de la documentación escrita desde simples hojas de permiso hasta folletos de hasta 28 páginas conteniendo múltiples testificaciones firmadas por el paciente y puestas en conocimiento de las partes interesadas.

Los problemas de la amplitud de la revelación y de la materialidad del riesgo son componentes clave para satisfacer los requirimientos legales del consentimiento informado, pero deben de ser evaluados originariamente en el contexto de una conversación.

Uno se pregunta si no sucederá que, en respuesta a las modificaciones de la doctrina legal, los médicos modifican sus formularios de consentimiento sin alterar la manera en la que se los presentan a los pacientes. Quizás el interrogante debería ser ¿cuántos médicos se preocupan de implicarse en mantener el intercambio oral de información cuando el formulario de consentimiento ya ha sido firmado?".
\end{abstract}

${ }^{1}$ Tomado de Vaccarino JM. Consent, informed consent and the consent form. N Engl J Med 1978; 298(8): 455. 
merece llamarse "paternalismo con formulario". Y éste es un esquema todavía extraordinariamente prevalente en nuestro medio sanitario.

MITO 3. La buena práctica clínica consiste en realizar bien los actos clínicos desde el punto de vista exclusivamente científico-técnico; el consentimiento informado no tiene nada que ver con esto.

Respuesta. Falso. La buena práctica clínica es la que actúa, desde el punto de vista jurídico, conforme a la lex artis. La visión tradicional reduce esta expresión al obrar correcto desde el punto de vista científico-técnico, pero la visión moderna -sustentada tanto en la legislación como en las decisiones judiciales- afirma que la buena práctica conforme a lex artis es algo más. Exige, además de la corrección técnica, la obtención del consentimiento informado.

Explicación. La visión tradicional de la lex artis es coherente con la cosmovisión paternalista. Como ya hemos visto, la corrección -tanto en sentido técnico como moral- del acto clínico era definida unilateralmente por los médicos y apoyada por jueces y legisladores. La voz del paciente era innecesaria. Esto ha cambiado radicalmente. Hoy en día, el buen cirujano -por poner un ejemplo- ya no es sólo un profesional que opera bien, sino que además proporciona a sus pacientes información de calidad, los implica en la toma de decisiones y obtiene un consentimiento válido. Este es el sentido auténtico del "Principio general 6" del artículo 2 de la Ley 41/2002: "Todo profesional que interviene en la actividad asistencial está obligado no sólo a la correcta prestación de sus técnicas, sino al cumplimiento de los deberes de información y de documentación clínica, y al respeto de las decisiones adoptadas libre y voluntariamente por el paciente".

Quien no lo haga así es un profesional mediocre desde el punto de vista ético y además incumple sus obligaciones legales.

MITO 4. El fundamento ético del consentimiento informado es el principio de autonomía.

Respuesta. Falso. El fundamento ético del consentimiento informado consiste en la articulación correcta de los cuatro principios clásicos de la bioética moderna (Tabla 2) en el marco de las relaciones clínicas.

Explicación. Aunque es verdad que, como hemos visto, el ariete que ha generado la crisis de la beneficencia paternalista tradicional ha sido la introducción de la idea de autonomía moral de las personas, es un error reducir a ello la fundamentación ética del nuevo modelo. De hecho, la mayor parte de la literatura norteamericana sobre consentimiento informado, incluidos los autores de prestigio -como los ya

Tabla 2. Los cuatro principios de la bioética.

\footnotetext{
Principio de No-Maleficencia

Debe evitarse realizar daño físico, psíquico o moral a las personas. Para ello debe evitarse realizarles intervenciones diagnósticas o terapéuticas incorrectas o contraindicadas desde el punto de vista científico-técnico y clínico, inseguras o sin evidencia suficiente, y proteger su integridad física y su intimidad.

Principio de Justicia

Debe procurarse un reparto equitativo de los beneficios y las cargas, facilitando un acceso no discriminatorio, adecuado y suficiente de las personas a los recursos disponibles, y un uso eficiente de los mismos.

Principio de Autonomía

Debe respetarse que las personas gestionen su propia vida y tomen decisiones respecto a su salud y su enfermedad.

Principio de Beneficencia

Debe promocionarse el bienestar de las personas, procurando que realicen su propio proyecto de vida y felicidad en la medida de lo posible.
} 
citados Faden, Beauchamp, Appelbaum, Lidz o Meisel- lo ha cometido.

Este tipo de fundamentación produce en los profesionales sanitarios la impresión de que, ahora, el que "toma el mando" en el nuevo modelo es, unilateralmente, el paciente. El médico queda relegado a mero ejecutor de los deseos del enfermo, que se fundamentan en sus derechos como usuario, y que son ilimitados. Pero eso no es así, ni siquiera desde la perspectiva del propio paciente que sigue esperando que sus médicos le ayuden a tomar decisiones, le recomienden, le sugieran, le acompañen, le expliquen, aunque respetando su autonomía última para aceptar o rechazar una intervención.

Por eso es necesario trabajar con otro modelo de fundamentación que articule los cuatro principios. Inspirándonos en Diego Gracia ${ }^{5}$ podemos decir que los cuatro principios no están situados todos en un mismo plano, sino en dos diferentes aunque complementarios (Figura 1).

- Un primer plano lo marcan los principios de no-maleficencia y justicia, y tiene que ver con el marco estructural en el que tiene lugar la relación clínica. El segundo plano establece los límites entre lo que puede o no puede decidirse. Así, lo primero que tienen que garantizar las organizaciones sanitarias y los profesionales es que la atención a los ciudadanos evite en lo posible el riesgo de producirles daño y ponga a su disposición los procedimientos y tecnologías más efectivas para la protección y promoción de la salud. Lo segundo que tienen que asegurar es que la inversión de los recursos públicos en dichos procedimientos y tecnologías sea eficiente y que, una vez se han puesto a disposición de los ciudadanos, el acceso a ellos sea equitativo.

- En un plano diferente se encuentran las obligaciones nacidas del contenido de la relación clínica. Aquí, el encuentro entre los profesionales y los pacientes permitirá concretar lo que se entienda por mejorar o cuidar la salud de las personas, y establecer, de entre las prácticas indicadas, las que se realizarán finalmente y las que no. El único límite es el que establecen, en el plano anterior, la no-maleficencia y la justicia.

De este modo, el profesional beneficente aportará a la relación clínica sus conocimientos científico-técnicos y su experien-

NO-MALEFICENCIA

\section{JUSTICIA}

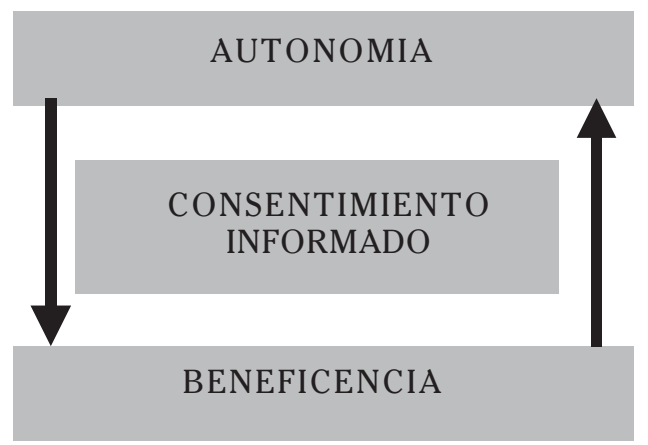

Figura 1. El Consentimiento Informado: Fundamentación ética. 
cia para establecer la indicación adecuada y el paciente autónomo aportará sus valores, sus preferencias y sus creencias.

Lo que "debe hacerse", por tanto, será el resultado de la ponderación prudente de las obligaciones derivadas del respeto a la autonomía y a la beneficencia, en un marco preestablecido por la no-maleficencia y la justicia. Un paciente no puede "exigir" determinadas actuaciones por muy autónomo que sea o porque "haya cotizado muchos años a la Seguridad Social". No puede solicitar que se le hagan intervenciones claramente maleficentes -por ser contrarias a la evidencia científica- o injustas, por suponer un despilfarro desde el punto de vista del análisis coste-beneficio.

MITO 5. La obtención del consentimiento informado es una obligación de los profesionales que realizan las intervenciones y los que las indican no tienen nada que ver con ello.

Respuesta. Falso. Es cierto que, según el artículo 8.1 de la Ley 41/2002, la obligación de obtener el consentimiento del paciente es del profesional que realiza la intervención -sea médico, enfermero o psicólogo clínico- y que éste además tiene que asegurarse de que el paciente ha recibido una información adecuada, pero esta responsabilidad de información es, según el artículo 4.3. de la misma Ley, compartida con todos los profesionales que intervienen en el proceso de atención. Cada uno de ellos participará según su grado de competencia, pero, en principio, nadie está eximido de esta obligación. De todos modos, el médico responsable del paciente tiene que actuar como garante de que el proceso informativo sea adecuado.

Explicación. Esta cuestión es muy importante porque ha generado frecuentes conflictos entre los profesionales y, lo que es peor, se ha incorporado al diseño o a la práctica habitual de muchos procesos asistenciales. Así, unas veces se ha defendido que el facultativo que indica una técnica no tiene obligación de informar y otras que los profesionales que la realizan pueden dar por supuesto que el paciente ha sido informado. Ninguna de esas dos posiciones es correcta: el derecho del paciente a aceptar o a rechazar una actuación tras haber sido debidamente informado, suscita obligaciones en todos los profesionales que participan en este proceso. El que alguno de los intervenientes no cumpla con su deber, no exime a los demás escudándose en que "era el otro el que tenía que haberlo hecho". Quien no asume su tarea de informar actúa de forma contraria a la ética profesional e incumple obligaciones jurídicas muy claras y estrictas.

Estos malentendidos son especialmente preocupantes en las intervenciones quirúrgicas. Es frecuente que la decisión de intervenir, la orden de ingreso y la entrega del formulario de consentimiento las realice un cirujano diferente del que efectuará la cirugía, a menudo sin que el propio paciente conozca tal eventualidad. De hecho, con frecuencia, la "firma" del formulario es un requisito administrativo para poder entrar en la lista de espera quirúrgica. Llegado el momento, no es raro que el cirujano que va a operar al paciente ni siquiera hable antes con él. De hecho, en ocasiones, lo verá por primera vez cuando éste ya esté dormido o premedicado. Asume así que el documento firmado da cuenta de que el proceso de consentimiento se efectuó correctamente y que él está protegido legalmente, pero este supuesto puede ser peligroso. En primer lugar porque el paciente autorizó la intervención al profesional con quien decidió ésta y no queda del todo claro que este consentimiento sea automáticamente extensible a cualquier otro cirujano. Es decir, la idea del "consentimiento" es la de una autorización que una persona da a otra para que ésta segunda realice algún acto que le afecta. Se desarrolla pues en un ámbito interpersonal y no meramente formal, estructural y organizativo. Primar lo segundo en detrimento de lo primero puede ser problemático. En segundo lugar porque el profesional que va a intervenirle presupone que el proceso de información fue correcto, pero esto puede no ser así. Por estos dos motivos lo más prudente sería que un cirujano que va a operar a un paciente al que no conoce, se asegure de que la información recibida por éste ha sido correcta, la ha entendido bien y acepta que sea él quien lo intervenga. Seguramente aplicar esta concepción exigiría modificar los pro- 
cesos organizativos de los servicios quirúrgicos en muchos hospitales. Ello no es una tarea fácil, ni algo que pueda acometerse de la noche a la mañana. Pero iniciar este cambio sería una clara expresión de compromiso organizacional con la calidad del proceso de consentimiento informado.

MITO 6. El consentimiento informado no tiene ningún sentido porque la mayoría de los pacientes no desean ser informados.

Respuesta. Falso. Los ciudadanos han dicho, a través de sus legisladores y gobernantes democráticamente elegidos, que desean que los profesionales sanitarios les ofrezcan información y les impliquen en el proceso de toma de decisiones. Y lo han dicho mediante la aprobación de leyes, como la Ley 41/2002 y todas las leyes autonómicas similares. Otra cosa es cómo cada ciudadano quiere que se dé contenido a este derecho, lo que obliga a los profesionales a explorar esta cuestión de forma personalizada en cada acto clínico.

Explicación. La práctica clínica diaria dice que es cierto que muchos pacientes se conforman con poca información y adoptan roles pasivos en el proceso de toma de decisiones. Pero los profesionales no deben presuponer por ello que si un paciente no demanda información es porque no la desea. La oferta explícita de información y la reiterada invitación a participar en el proceso de toma de decisiones son obligaciones éticas y jurídicas de los profesionales. Así lo establece el artículo 5.c de la Ley 44/2003 de Ordenación de las Profesiones Sanitarias ${ }^{6}$.

El paciente puede ejercer su autonomía moral de una forma particular: mediante el rechazo de la información y el deseo de que sea el médico el que decida. Este es un derecho reconocido por el artículo $4.1 \mathrm{de}$ la Ley 41/2002, pero esto no exime al profesional de tener que ofrecer explícitamente la información al paciente.

De todas formas, la experiencia dice que la actitud hacia la información y la participación por parte de los pacientes varía dependiendo de la edad y el estatus socioeconómico o cultural. Las generaciones jóvenes son cada vez más conscientes de sus derechos y quieren participar más, deliberar más, decidir más sobre su salud.
Desean que los profesionales se impliquen con ellos en ese proceso porque necesitan que les ayuden con su punto de vista técnico y humano. No quieren ni el puro paternalismo de antaño, ni la frialdad burocrática, ni la información descarnada sin acogida personal, ni posiblemente tampoco quieren que los profesionales sean sus "amigos". Quieren más bien compañeros de viaje, consejeros que les ayuden a interpretar sus propios deseos, necesidades y valores, y que les ayuden a decidir libre e informadamente ${ }^{7}$.

De hecho, los pacientes van a necesitar cada vez menos de los profesionales para obtener información científica porque Internet ya la está ofreciendo, bien a través de las páginas de salud o a través de las comunidades virtuales de pacientes o familiares de pacientes que pueden gestionar de forma no-profesional información de alta calidad científico-técnica ${ }^{8}$. Así pues, el papel clave del profesional sanitario del futuro no será el de procurar información científica, sino el de dar consejo y apoyo en el proceso de toma de decisiones clínicas $^{9}$.

MITO 7. Los pacientes tienen derecho a rechazar un tratamiento, incluso si con ello ponen en peligro su salud o su vida, pero sólo antes de que se les aplique. Una vez iniciado éste los profesionales no pueden retirarlo.

Respuesta. Falso. Es un principio ético y jurídico básico de la teoría del consentimiento informado que las decisiones autónomas de los pacientes deben respetarse. Decisiones autónomas son aquellas que las personas toman de forma libre, informada y capaz. Estas decisiones pueden ser de aceptación o rechazo de cualquier intervención diagnóstica o terapéutica, y en el caso de que la decisión inicial sea de aceptación, posteriormente el paciente puede revocar libremente ese consentimiento, siempre y cuando asuma de forma libre e informada las consecuencias de su revocación.

Explicación. La negativa de los profesionales sanitarios, e incluso de los jueces, a aceptar la libertad de los pacientes informados y capaces para rechazar tratamientos o revocar su previo consentimiento 
para recibirlos es una expresión muy clara de lo difícil que resulta superar la mentalidad paternalista. Y ello a pesar de que la Ley 41/2002 establece de forma clara y contundente este principio jurídico en sus artículos 2.3, 2.4 y 8.5.

Lo cierto es que cuando un paciente decide libremente asumir la posibilidad de lesión de su salud o de su vida y rechazar un tratamiento que le resulta inadmisible, con demasiada frecuencia a todos los implicados les asaltan de nuevo los fantasmas paternalistas de la prioridad absoluta e indiscriminada de la protección de la vida sobre el ejercicio de la libertad personal. Testigos de Jehová adultos que rechazan transfusiones sanguíneas, pacientes renales no trasplantables y en diálisis que solicitan la suspensión de la misma, pacientes tetrapléjicos, con ELA o con distrofias musculares que solicitan la desconexión de respiradores o incluso presos en huelga de hambre, son ejemplos claros y polémicos de este debate.

La idea de que, una vez iniciada una tecnología ya no puede ser retirada bajo ningún concepto aunque haya dejado de ser útil o el paciente capaz la rechace, es cuestionable ética y jurídicamente. Es más bien la idea contraria la adecuada: es éticamente obligatorio y jurídicamente correcto retirar terapias inútiles o que el paciente capaz rechaza. Y ello aun cuando de dicha retirada se derivara la muerte del paciente. De no ser así, ¿qué sentido tiene que aceptemos que los ciudadanos tomen estas decisiones para cuando sean incapaces, mediante el documento de voluntad anticipada, si rechazamos que las tomen cuando todavía son capaces?, ¿tienen los pacientes incapaces más derechos que los capaces?

MITO 8. La evaluación de la capacidad de los pacientes para decidir es responsabilidad de los psiquiatras.

Respuesta. Falso. El médico que atiende al paciente es el responsable de evaluar y dictaminar la capacidad de éste para tomar decisiones. Así lo establecen -por primera vez de forma explícita en la historia de nuestro ordenamiento jurídico- los artículos 5.3 y 9.3.a de la Ley 41/2002. No es responsabilidad ni de los psiquiatras ni del personal de enfermería. Otra cosa es que el médico responsable solicite a un psiquiatra su opinión al respecto, esto sería un peritaje de capacidad.

Explicación. La evaluación de la capacidad de los pacientes es, sin lugar a dudas, la cuestión más compleja y peor resuelta en la práctica de la teoría del consentimiento informado. Cuando un clínico se pregunta por la manera de evaluar la capacidad de un paciente, instintivamente vuelve su cabeza hacia las leyes. La desagradable sorpresa es que el Derecho no concreta nada a este respecto. A lo sumo dice que un paciente es capaz cuando tiene "entendimiento" y "voluntad" suficientes, pero no establece ningún criterio objetivo ni ninguna forma práctica de medición de tales cosas.

Cuando un juez quiere aclarar tales extremos en un caso concreto, llama a expertos -médicos forenses o psiquiatras forenses- para que hagan un peritaje. Lo sorprendente es que estos especialistas tampoco cuentan con ningún procedimiento estandarizado (al menos aplicable a un contexto clínico de toma de decisiones). Sus evaluaciones sobre la capacidad suelen ser el resultado de una combinación de herramientas -tests, escalas, etc. - habitualmente diseñadas para otras funciones y una evaluación clínica subjetiva mediante exploración y entrevista. Por su parte, los notarios, que también pueden y deben según el Código civil hacer tales valoraciones, tampoco tienen un procedimiento estandarizado.

Los clínicos españoles que llevan décadas haciendo "evaluaciones" de facto de la capacidad de los pacientes, lo hacen utilizando una mezcla de sentido común y experiencia profesional que, si bien sirve para salir indemne del trance, no puede sostenerse mucho tiempo más.

La única posibilidad es mirar lo que otros han hecho y plantearse la necesaria validación -rigurosa, conceptual y transcultural- de sus herramientas. Éstas se han desarrollado mayoritariamente en países anglosajones (Tabla 3) y la que, según las diferentes revisiones publicadas ${ }^{10-12}$, resulta de mayor sensibilidad y especifici- 
Tabla 3. Protocolos de evaluación de la capacidad.

\begin{tabular}{|c|c|c|c|c|c|c|}
\hline DENOMINACIÓN & ACRÓNIMO & AUTOR & PAÍS & AÑO & TIPO & PUNTÚA \\
\hline Aid to Capacity Evaluation & ACE & Etchells y col ${ }^{1}$ & Canada & 1999 & Entrevista semiestructurada & SÍ \\
\hline Capacity Assessment Tool & CAT & Carney y col ${ }^{2}$ & EE.UU. & 2001 & Entrevista estructurada & SÍ \\
\hline Capacity to Consent to Treatment Instrument & CCTI & Marson y col ${ }^{3}$ & EE.UU. & 1995 & 2 escenarios y entrevista & Sí \\
\hline Decision Assessment Meausure & DAM & Wong y $\operatorname{col}^{4}$ & UK & 2000 & Entrevista semiestructurada & Sí \\
\hline Hopemont Capacity Assessment Interview & HCAI & Edelstein ${ }^{5}$ & EE.UU. & 1999 & 2 escenarios y entrevista & Sí \\
\hline MacArthur Competence Assessment Tool & $\begin{array}{l}\text { MacCAT-T * } \\
\text { MacCAT-CR } \\
\text { MacCAT-CA }\end{array}$ & $\begin{array}{l}\text { Grisso \& } \\
\text { Appelbaum }^{6,7}\end{array}$ & EE.UU. & 1998 & $\begin{array}{l}\text { Entrevista semiestructurada } \\
\text { (1 escenario en la version CR) }\end{array}$ & Sí \\
\hline
\end{tabular}

* El McCAT existe en tres versiones "T" (Treatment) para decisiones clínicas de tratamiento, "CR" (Clinical Research) para participar en proyectos de investigación y "CA" (Criminal Adjudication) para imputabilidad.

1 Etchells E, Darzins P, Silberfeld M, Singer PA, McKenny J, Naglie G, et al. Assessment of patients capacity to consent to treatment. Journal of General Internal Medicine 1999;14:27-34. El protocolo está disponible en http://www.utoronto.ca/icb/disclaimers/ace.htm (Visitada el 15 de mayo de 2006)

2 Carney MT, Neugroschl J, Morrison RS, Marin D, Siu AL The development and piloting of a capacity assessment tool. The Journal of Clinical Ethics 2001;12:17-23.

3 Marson, DCCody, HA, Ingram KK, Harrell LE. Neuropsychological predictors of competency in Alzheimer's disease using a rational reasons legal standard. Archives of Neurology 1995;52:955-959.

4 Wong JG, Clare ICH, Holland AJ, Watson PC, Gunn M. The capacity of people with a mental disability to make a health care decision. Psychological Medicine 2000;30:295-306

5 Edelstein B. Hopemont capacity assessment interview manual and scoring guide. Morgantown, WV: West Virginia University, 1999.

6 Grisso T., Applebaum PS. Assessing competence to consent to treatment. New York: Oxford University Press, 1998.

7 Appelbaum PS, Grisso T. MacCAT-CR. Mac Arthur Competence Assessment Tool for Clinical Research. Sarasota (FL - USA):Professional Resource Press;2001.

dad es la MacArthur Competence Assessment Tool (MacCAT).

MITO 9. El respeto a las exigencias del consentimiento informado es un deber ético y jurídico de los profesionales que pueden satisfacerse sin que las organizaciones sanitarias inviertan recursos adicionales en ello.

Respuesta. Falso. La idea de que todo esto puede hacerse a "coste cero" es un mito muy difundido entre los gestores sanitarios. Con frecuencia pone de manifiesto un estilo de gestión más centrado en los resultados económicos que en la gestión de los valores que la organización dice defender. El respeto a los derechos de los pacientes en general y al consentimiento informado en particular requiere inversiones en formación de los profesionales, adecuación de los espacios físicos para facilitar una adecuada comunicación, diseño de procesos clínicos que incluyan explícitamente el consentimiento informado como algo esencial y, sobre todo, aumento del tiempo de dedicación de los profesionales a esta tarea. Todo ello implica un consumo de recursos que puede ser tan efectivo y eficiente como el destinado a otro tipo de actividades. Y es un indicador excelente de la seriedad del compromiso ético de la organización ${ }^{13}$.

Explicación. El fin último de las organizaciones sanitarias públicas no es la rentabilidad económica, sino la protección de la salud de la ciudadanía ${ }^{14}$. Como hemos visto, una visión moderna de este objetivo exige incorporar la voz de los usuarios y el respeto a sus derechos. Por eso, la inversión económica en planes y programas destinados a ello es socialmente efectiva y éticamente coherente.

Uno de los recursos más escasos de nuestro sistema sanitario es el tiempo. Es verdad que puede haber profesionales que no lo gestionen correctamente, lo pierdan o incluso algunos, sencillamente, no lo utilicen por incumplimiento del horario de trabajo o por absentismo laboral. Pero, seguramente, en muchas otras ocasiones la falta de tiempo es producto de una orga- 
nización de la asistencia que prioriza la producción en detrimento de la calidad. Quizás en la atención primaria es donde este fenómeno resulta más evidente y, cuando no hay tiempo, una de las cosas que se resiente es la calidad del proceso de consentimiento informado.

En el estudio de la Fundación Biblioteca Josep Laporte "Confianza en el Sistema Nacional de Salud 2006", los pacientes encuestados señalan expresamente como una necesidad relevante la de disponer de más tiempo para hablar con sus médicos ${ }^{15}$. Según Jadad, los pacientes -además de una elevada calidad científico-técnicaesperan de sus médicos básicamente cinco cosas: contacto visual directo, corresponsabilidad en la toma de decisiones, comunicación fluida y de calidad, accesibilidad a la consulta en un plazo de tiempo razonable y tiempo suficiente para explicarles sus necesidades, dudas, etc, y para que sus médicos les respondan adecuadamente $^{16}$.

MITO 10. La obtención del consentimiento informado es una responsabilidad de los médicos, pero no tiene nada que ver con la enfermería.

Respuesta. Falso. Este modelo de relación clínica afecta a todas las profesiones sanitarias que interactúan con pacientes o usuarios. Los actos clínicos enfermeros, de cuidado o de tratamiento, requieren la obtención previa del consentimiento informado.

Explicación. Es cierto que la visión clásica de la teoría del consentimiento informado y su desarrollo histórico, centrado demasiadas veces en los "papeles firmados" y en los "casos judiciales", han hecho creer a muchos profesionales sanitarios "no-médicos" que esta cuestión no les afectaba, pero están en un error. En el caso concreto de la enfermería, el Código Deontológico de la Enfermería española de 1989 recoge esta obligación de forma muy amplia $^{17}$. Y lo mismo puede decirse del artículo 5 de la Ley 44/2003 de Ordenación de las Profesiones Sanitarias que afecta a todos.

Resulta por tanto urgente que la enfermería sustituya su tradicional "maternalismo" por una relación con el paciente basa- da en la idea de consentimiento informa$\mathrm{do}^{18}$, de lo contrario, su actuación sería de muy baja calidad, y estaría incumpliendo sus obligaciones éticas y jurídicas.

\section{CONCLUSIÓN}

La desmitificación es uno de los requisitos indispensable para cualquier pensamiento crítico. Desvelar el discurso oculto en estos mitos, transmitidos de profesional a profesional en las reuniones informales o en las sesiones clínicas, es imprescindible para poder cambiar las prácticas reales en nuestras organizaciones sanitarias. En esta ocasión hemos afrontado diez mitos en torno a la teoría y práctica del consentimiento informado. No son los únicos, pero sí quizás los más importantes. Esperamos contribuir con ello a que el necesario cambio se haga realidad.

\section{BIBLIOGRAFIA}

1. FAdEN R, Beauchamp TL. A History and a Theory of Informed Consent. Neew York: Oxford University Press 1986: 100-101.

2. Simón P. El consentimiento informado: historia, teoría y práctica. Madrid: Triacastela 2000.

3. Boletín Oficial del Estado. Ley 41/2002, básica reguladora de la autonomía del paciente y de derechos y obligaciones en materia de información y documentación clínica. BOE núm 274, de 15 de noviembre de 2002. pp. 40126-40132.

4. LiDz CW, AppelBaum PS, Miesel A. Two models of implementing informed consent. Arch Inter Med 1988; 148: 1385-1389.

5. Gracia D, Ética Médica. En: FARreras P, RozMAN C, editores. Medicina Interna. Vol I. $3^{\text {a }}$ ed. Barcelona: Doyma 1995: 33-39.

6. Boletín Oficial del Estado. Ley 44/2003, de 21 de noviembre, de Ordenación de las Profesiones Sanitarias. BOE núm. 280, de 22 de noviembre de 2003. pp. 41442-41458.

7. Emanuel EJ, Emanuel LL. Four models of the patien-physician relationship. JAMA 1992; 267 (16): 2221-2226. (Traducido en: Emanuel EJ, Emanuel LL. Cuatro modelos de la relación médico-paciente. Cruceiro A, editora. Bioética para clínicos. Madrid: Triacastela; 1999. p. 109-126).

8. Esquivel A, Meric-Bernstam F, Bernstam EV. Accuracy and self correction of information 
received from an internet breast cancer list: content analysis. BMJ 2006; 332: 939-942.

9. JadAd AR, Enkin MW, Glouberman S, Groff P, STERN A. Are virtual communities good for our health? BMJ 2006; 332: 925-926.

10. StURman ED. The capacity to consent to treatment and research: A review of standardized assessment tools. Clin Psychol Rev 2005; 25: 954-974.

11. Moyce J, Gurrera RJ, Karel MJ, Eldestein B, O'ConNell C. Empirical advances in the assessment of the capacity to consent to medical treatment. Clinical implications and research needs. Clin Psychol Rev: En prensa.

12. Dunn LB, Nowrangi MA, Palmer BW, Jeste DV, SAKS ER. Assessing decisional capacity for clinical research or treatment: A review of instruments. Am J Psychiatry 2006; 163: 1323-1334.

13. SIMÓN P. La ética de las organizaciones sanitarias: el segundo estadio de desarrollo de la bioética. Rev Calidad Asistencial 2002; 17: 247-259.

14. Cortina A, Conill J. Ética, empresa y organizaciones sanitarias. En: Simón P, editor. Ética de las organizaciones sanitarias. Madrid: Triacastella 2005: 15-32.

15. Jovell AJ, ed. Confianza en el Sistema Nacional de Salud. Resultados del estudio español. Barcelona: Fundació Biblioteca Josep Laporte, 2006. Disponible en http://www.fbjoseplaporte.org (Visitada el 1 de noviembre de 2006).

16. JADAD AR, RIZO CA, ENKIN MW. I am a good patient, relieve it or not. BMJ 2003; 326: 1293-1295.

17. Organización Colegial de Enfermería. Código Deontológico de la enfermería española. Madrid: Consejo General de Colegios de Diplomados de Enfermería de España, 1989.

18. SIMÓN P, BARRIO IM. El consentimiento informado en enfermería: un modelo integral. JANO 1995; 48: 911-921. 